\title{
Kloster Murrhardt und die Reformation unter besonderer Berücksichtigung der Murrhardter Besitzungen in Schwäbisch Hall
}

\author{
von GERHARD Fritz
}

\section{Die Lage in vorreformatorischer Zeit}

Die Geschichte des Klosters Murrhardt fügt sich bis weit in die 1520er Jahre hinein nicht in die Chronologie der gesamtdeutschen oder auch nur der württembergischen Reformation ein. Innerkatholische Versuche, den Vorschriften der alten Benediktinerregel zu entgehen, daraus sich ergebende vorlutherische Reformbestrebungen, dauernde Finanznöte und ständige Versuche, aus dem Wust von geistlicher Reformbedürftigkeit und finanziellen Engpässen herauszukommen, vermengen sich mit den spezifisch württembergischen Turbulenzen um die Vertreibung Herzog Ulrichs zu einem unentwirrbaren Dickicht. Während ganz Deutschland seit 1517/18 gebannt auf Martin Luther schaute, steckte Murrhardt in eben diesem, für das Kloster unüberschaubaren Dickicht mitten drin, und man fragt sich, wie viel Zeit und Aufmerksamkeit denn die Murrhardter Mönche angesichts ihrer existenziellen Nöte für den Mann aus Wittenberg haben konnten. Als Martin Luther 1517 mit seinen Thesen an die Öffentlichkeit trat, konnte das Benediktinerkloster Murrhardt auf eine mindestens 700 Jahre alte Geschichte zurückblicken. Die letzten Jahrzehnte dieser Geschichte waren für das Kloster turbulent und von Reformen geprägt gewesen, die mit Luther gar nichts zu tun hatten, sondern sich noch völlig im Rahmen der zahlreichen innerkatholischen Reformbestrebungen bewegten. Noch unter dem Abt Johannes Schradin (14861501) hatte sich das Kloster durch Baumaßnahmen - hauptsächlich die Vergrößerung und Befestigung der kombinierten Stadt- und Klosteranlage sowie die Anlage großer künstlicher Seen - zudem die Anschaffung von Kunstwerken offenbar finanziell übernommen. ${ }^{1}$ Das in der Zeit nach Schradin unter dem schwachen, durch Krankheit und Erblindung wenig handlungsfähigen Abt Lorenz Gaul (1501-1508) ziemlich heruntergekommene Kloster sollte nach Wunsch des zuständigen Landesherrn - des Herzogs Ulrich von Württemberg - und des zuständigen Würzburger Diözesanbischofs - Lorenz von Bibra - 1507 dringend reformiert werden. Die Murrhardter Mönche hatten freilich andere Vorstellun-

1 Vgl. zu Schradin allgemein: Gerhard Fritz: Stadt und Kloster Murrhardt im Spätmittelalter und in der Reformationszeit (FWFr 34). Sigmaringen 1990, S. 56-64 und 342 f. 
gen und wollten ihr Kloster in ein weltliches Stift umwandeln. Vorbilder hatte es genug gegeben: Im benachbarten Backnang war 1477 das regulierte Augustiner-Chorherrenstift in ein weltliches Stift umgewandelt worden, und in Ellwangen und Komburg - beides Benediktinerklöster wie Murrhardt - hatte man dasselbe 1488 und 1460 getan, und das Kloster Selz im Elsass hatte sich 1481 ebenfalls entsprechend umgewandelt - übrigens unter einem Abt, der zuvor Mönch in Murrhardt gewesen war. Der Haller Priester und Notar Georg Widman, der selbst jahrzehntelang für das Kloster in Murrhardt tätig war und dort eine Klosterchronik verfasst hat, sah ein ganz simples Motiv für die Murrhardter Umwandlungsbestrebungen, die sich von 1507 bis 1509 hinzogen: ${ }^{2}$ Die der Lasten ihrer Ordensregel überdrüssigen Murrhardter Mönche hätten überlegt, wie sie aus der kutten kommen könnten. ${ }^{3}$ Nach einigem Hin und Her erklärten sich der Herzog und der Bischof mit dem Umwandlungswunsch einverstanden und erlaubten, dass die Murrhardter sich in Rom die erforderliche päpstliche Zustimmung holten.

Das Unternehmen ging, anders als in Backnang, Komburg, Ellwangen und Selz, völlig schief: Die Murrhardter Delegation, die man wegen der päpstlichen Umwandlungsbulle nach Rom geschickt hatte, brachte es fertig, die reich gefüllte Reisekasse und das Umwandlungsgeld, das für den Heiligen Stuhl vorgesehen war, bei ihrem offenbar recht komfortablen Aufenthalt in Rom zu verjubeln. Nur mit einem Kredit des Bankhauses Fugger wurden die Murrhardter wieder handlungsfähig - allein: Fugger behielt die Bulle ein und wollte sie nur gegen Rückzahlung des Kredits wieder herausgeben. Als Herzog Ulrich das herausbekam, und als er auch noch sah, dass die Bulle nicht nach seinem Sinne ausgefallen war, ließ er die mittlerweile zurückgekehrte Murrhardter Romgesandtschaft erst einmal einsperren und verbot die Umwandlung des Klosters in ein weltliches Stift. Stattdessen ordnete er eine strenge Klosterreform im Rahmen der alten benediktinischen Regeln an.

Tatsächlich musste das Kloster sich 1510/11 eine harte Reform gefallen lassen, bei der das Klosterpersonal teilweise ausgetauscht und durch Lorcher Mönche ersetzt wurde. Weil sich insbesondere in finanzieller Hinsicht kaum etwas bewegte, wurden 1512 die Zügel noch mehr angezogen: Um die klösterlichen Ausgaben radikal zu drosseln, wurden dem Kloster harte Sparmaßnahmen auferlegt. Diese gipfelten um die Jahreswende 1517/18 in der ultimativen Maßnahme zur Kostenreduzierung, nämlich in der Zwangsexilierung des gesamten Konvents, der in anderen Klöstern untergebracht wurde. Zwei Laien versuchten derweil, die Finanzen des Klosters in Ordnung zu bringen - allerdings vergeblich. Ende 1518 verweigerte sich ein vom Landkapitel der württembergischen Benediktiner

2 Ebd., S. $64-80$ und $343 \mathrm{f}$.

3 Widmans Chronica. Bearb. v. Christian Kolb (Württembergische Geschichtsquellen $6=$ Geschichtsquellen der Stadt Hall 2). Stuttgart 1904, S. 138 f.; vgl. auch: Ders.: Des Haller Chronisten Georg Widman Leben. In: WFr NF 6 (1897), S. 21-43. 
als Krisenmanager herbeigerufener Abt aus Erfurt, weil ihm die Anreise zu weit war. Die Visitationsgewalt wurde daraufhin dem Abt von Hirsau übergeben. ${ }^{4}$ Ohne dass sich Grundlegendes gebessert hatte, kehrten die Murrhardter Mönche Anfang 1519 wieder in ihr Kloster zurück. All dies fiel zeitlich mit den dramatischen Vorgängen rund um Martin Luther und mit der Vertreibung Herzog Ulrichs aus seinem Land durch den Schwäbischen Bund zusammen. Nachdem der Bund 1520 Württemberg an die Habsburger übergeben hatte, spielte sich bis 1534 die gesamte Klostergeschichte unter habsburgischer Herrschaft ab.

\section{Das Kloster zwischen 1519 und 1534}

Das bisher Dargestellte zeigt, wie wenig die damals und heute allerorten erörterten Ereignisse rund um Luther in den Jahren 1517-1519 die Abläufe in Murrhardt prägten. In Murrhardt hatte das Kloster ganz andere Sorgen, und die waren - neben dem zweifellos zumindest zeitweise auch vorhandenen geistlich-moralischen Tiefstand - zunächst einmal finanzieller Art. Die politischen Verwerfungen um Herzog Ulrich und die Machtübergabe an die Habsburger dürften die Aufmerksamkeit der Murrhardter Mönche mindestens ebenso beansprucht haben wie die Nachrichten von dem kometenhaft berühmt werdenden Luther.

Erstaunlicherweise arbeitete das Kloster seit 1519 mit einigem Erfolg daran, aus der Finanzkrise herauszukommen. Dafür war nicht der wieder aus Lorch nach Murrhardt zurückgekehrte Abt Oswald Binder verantwortlich. Seine Stärken lagen weniger in der Verwaltung, sondern im geistlichen Bereich. Widman schreibt von ihm, er sei ein frommer, geisztlicher, andächtiger gottesfürchtiger mann [gewesen], aber in burgerlichen sachen undt hauszhaltung nicht gantz lauffenlich. Vielmehr war der eigentliche Sanierer der Großkeller Martin Mörlin, nach Widman ein lauffenlicher, geschickter mensch, der von allen männiglich geliebth undt wert. Mörlin sollte nach Binders Tod 1527 seit 1528 dessen Nachfolger als Abt werden. Am Vorabend des Bauernkriegs 1525, so Widman, sei der Zustand des Klosters wieder leidentlich gewesen. Die Sanierungsbemühungen des Klosters erlitten mit dessen Plünderung (und dem Verlust der gesamten Verwaltungsunterlagen und des Klosterarchivs) durch die Bauern dann aber einen schweren Rückschlag. Nach dem Ende des Bauernkriegs versuchte das Kloster zwar für erlittene Schäden die Bauern haftbar zu machen und diese zum Schadenersatz heranzuziehen. Dies dürfte aber nur bedingt erfolgreich gewesen sein. ${ }^{5}$ Auch Streitigkeiten zwischen Murrhardt und den Schenken von Limpurg um

4 HStAS A 508, Bü 2: Schreiben von Abt Johann von St. Peter zu Erfurt vom 25. Dezember 1518. 5 Widman (wie Anm. 3), S. 140; vgl. auch Fritz 1990 (wie Anm. 1), S. 81 ff., 94 ff. Zusätzlich zu den bei Fritz genannten Verkäufen zwischen 1519 und 1525 wäre noch anzufügen der Verkauf der murrhardtischen Hälfte am großen und kleinen Zehnt zu Schwarzenweiler ob Forchtenberg an Hohenlohe um 80 fl rh (HStAS A 508, Bü 17, Papier, ohne Siegel, zeitgleiche Kopie). 
Strafzahlungen der Bauern in dem Kondominatsort Ottendorf führten zu nichts. ${ }^{6}$ Martin Mörlin versuchte mit gemischtem Erfolg hier und in anderen Fällen hartnäckig Rechtstitel des Klosters geltend zu machen. ${ }^{7}$

Als existenziell gefährlich für alle württembergischen Klöster erwies sich das Verhalten der württembergischen Ehrbarkeit auf den Landtagsberatungen in Tübingen im Oktober/November 1525. Erzherzog Ferdinand, der Bruder Kaiser Karls V. und seit 1520 Landesherr in Württemberg, hatte den württembergischen Ämtern insgesamt die Schuld am Bauernkrieg angelastet und verlangte eine kollektive Entschädigung von allen Ämtern. Um ihre Haut zu retten, waren die Vertreter der weltlichen Ämter auf dem Landtag ohne Weiteres bereit, zur Begleichung der habsburgischen Forderungen die Klöster zu opfern. Die Einziehung aller Klöster hätte die habsburgischen Forderungen weithin befriedigt. AuBerdem war - bedingt durch Luther und den Bauernkrieg - die Stimmung in der Bevölkerung sowieso weithin antiklösterlich, so dass die Säkularisierung der Klöster der immer noch unruhigen Stimmung unter den Bauern einiges an Schärfe genommen hätte. Schließlich und endlich hätte die Position der weltlichen Ämter auf dem Landtag einen deutlichen Machtzuwachs erfahren, wenn die Klöster in Gestalt ihrer Äbte dort nicht mehr vertreten gewesen wären.

Hätte der Erzherzog sich nicht energisch für den Erhalt der Klöster ausgesprochen, hätte deren Existenz angesichts der Stimmung auf dem Landtag wohl schon Ende 1525 geendet. So blieben sie zwar erhalten, mussten von den habsburgischen Geldforderungen von $106000 \mathrm{fl}$ aber angesichts ihrer im Vergleich zu den weltlichen Ämtern erheblich geringeren Wirtschaftsleistung den weit überproportionalen Anteil von $36000 \mathrm{fl}$ übernehmen.

Zur Ermittlung der Kostenverteilung wurde noch 1525 in Württemberg die Zählung und finanzielle Schätzung aller Herdstätten durchgeführt. Die Herdstättenlisten sind im Großen und Ganzen bis heute erhalten geblieben und bilden eine der wichtigsten flächendeckend vorhandenen seriellen Quellen zur Sozial- und Wirtschaftsgeschichte des Landes. Murrhardt erwies sich unter den 1525 erfassten 13 württembergischen Mannsklöstern zusammen mit St. Georgen als das finanzschwächste. Es wurde mit $350 \mathrm{fl} \mathrm{veranschlagt.}{ }^{8}$

Damit nicht genug: Für den Abwehrkampf gegen die Türken - sie näherten sich Wien, das sie schließlich wenige Jahre später, 1529, (erfolglos) belagerten stellte Württemberg im September 1526 insgesamt 2000 Fußknechte. Zur Finanzierung wurde eine Türkensteuer ausgeschrieben. Der Murrhardter Anteil betrug $111 \frac{1}{2} \mathrm{fl}$. Es ist allerdings fraglich, ob Murrhardt - wohl wegen völliger Zah-

6 HStAS A 54, Bü 25, Schreiben von Schenk Georg von Limpurg an Erzherzog Ferdinand vom 30. August 1526 (Do. n. Bartholomäi) 1526. Murrhardt und Limpurg wollten sich auf einem gütlichen Tag in Lorch einigen; vgl. auch StAL B 114, Bü 1749-9, Schreiben von Abt Oswald von Murrhardt vom 13. Mai (Mo. n. Jubilate) 1527 an Schenk Georg.

7 Vgl. die umfangreichen Akten von 1529 zum Streit zwischen Murrhardt und dem Komtur von Rohrdorf um den Zehnt in Winzerhausen (HStAS A 508, Bü 3 und U 21).

8 Fritz 1990 (wie Anm. 1), S. 94 f. 
lungsunfähigkeit - diesen Betrag zahlen musste, denn es fehlt in der abschließenden Zahlungsliste der württembergischen Mannsklöster. ${ }^{9}$ Bei der nächsten Türkensteuer 1529 war es mit 28 fl veranschlagt. ${ }^{10}$ An den Türkensteuern sollte sich auch in den folgenden Jahrzehnten - also nach der Reformation des Klosters - nichts ändern. Weitere Türkensteuern folgten 1542 und 1545 und - als Dauerzustand - auch in nachreformatorischer Zeit. Deren Listen sind - anders als 1526 und 1529 - erhalten geblieben und stellen ebenfalls wichtige sozialgeschichtliche Quellen dar. Von dem offenbar regelmäßig jedes Jahr zu bezahlenden Murrhardter Türkensteuer-Anteil ist von 1568/69 der Nachweis in der einzigen erhalten gebliebenen klösterlichen Amtsrechnung des 16. Jahrhunderts vorhanden. ${ }^{11}$ Insgesamt waren die Jahre bis 1534 die letzte Zeit, in der - allen lutherischen Turbulenzen im Reich zum Trotz - die württembergischen Klöster noch einmal geordnete Verhältnisse hatten. Solange sie unter habsburgischem Schirm standen, war ihr Fortbestand gesichert. Mit der Rückkehr von Herzog Ulrich aus dem hessischen Exil 1534 und mit der erfolgreichen Wiedereroberung seines Landes änderten sich die Verhältnisse schlagartig. Ulrich bekam bekanntlich sein Herzogtum wieder zurück, wenn auch nur als habsburgisches Afterlehen. Die im Zusammenhang mit der Wiedergewinnung des Landes entstandenen immensen Kosten legte der Herzog auf die Ämter um, d. h. auf die Klöster kamen erneut hohe Kosten zu.

Hier ist ein Blick über die Jahre 1534/35 hinaus wichtig, denn die $60000 \mathrm{fl}$ Kriegskosten für die Wiedereroberung des Landes waren nur der Auftakt zu Schlimmerem. Als Ulrich 1546 im Schmalkaldischen Krieg auf der falschen Seite gestanden hatte und gegen Kaiser Karl V. unterlag, waren im Januar 1547 schwindelerregende 300000 fl fällig, um überhaupt nur einen Waffenstillstand erreichen zu können. ${ }^{12}$ Über Ulrich schwebte trotz des Waffenstillstandes weiterhin das Risiko des Felonieprozesses: Ulrich hatte als seit 1535 habsburgischer Lehensmann mit dem Krieg gegen den Kaiser seinen Lehenseid gebrochen - und das konnte in der vollständigen Entziehung des Herzogtums Württemberg als habsburgisches Afterlehen enden. Die Verwerfungen Ulrichs hatten auch für seine Nachfolger endlose finanzielle Belastungen zur Folge. Herzog Christoph konnte sich dem Felonieprozess nur entziehen und wieder ein geordnetes Verhältnis zum Reichsoberhaupt aufbauen, indem er 1552 weitere $300000 \mathrm{fl}$ an die Habsburger zahlte. ${ }^{13}$ Noch Christophs Nach-Nachfolger Herzog Friedrich hatte,

9 HStAS L D (Tomi actorum, Tomus Austriacus 3, S. 1301-1305 und 1340).

10 Ebd., S. $1567 \mathrm{ff}$.

11 HStAS A 303, Bd 10110, künftig in einer vom Vf. vorbereiteten elektronischen Edition.

12 Martin Brecht, Hermann Ehmer: Südwestdeutsche Reformationsgeschichte. Stuttgart 1984, S. 307.

13 Hans-Martin Maurer: Herzog Christoph. In: Robert Uhland (Hg.): 900 Jahre Haus Württemberg. Stuttgart u. a. 1984, S. 136-162, hier 143; auch: Mathias Langensteiner: Herzog Christoph. In: Christoph 1515-1568. Ein Renaissancefürst im Zeitalter der Reformation. Begleitband zur Ausstellung im Landesmuseum Württemberg 24. Oktober 2015 bis 3. April 2016, S. 15-34, hier 20. 
um das Afterlehensverhältnis zum Hause Habsburg abzulösen, 1599 die noch höhere Summe von $400000 \mathrm{fl}$ zu zahlen. ${ }^{14}$

Aber was bedeuteten diese Summen für den einfachen Mann und für das Kloster Murrhardt? Die aus der Politik Ulrichs resultierenden Staatsschulden sollten auf viele Jahrzehnte und damit weit in die Zeit des evangelischen Murrhardt der größte Ausgabenposten in der Rechnung des Klosteramts überhaupt werden. Von Gesamtausgaben von $5978 \mathrm{lb} \mathrm{h}$ - die Naturalausgaben bleiben hier außerhalb der Betrachtung - entfielen 1568/69 allein $1890 \mathrm{lb}$ oder 31,6\% auf die $a b$ lösungshilff des Herzogs, also auf die Bedienung der Staatsschulden. ${ }^{15}$

\section{Von der ersten Reformation unter Herzog Ulrich 1534/35 zum Interim 1548-1552}

Aber zurück in die 1530er Jahre. Auch im Kloster Murrhardt wurde 1534/35 zügig jeder katholische Ritus abgeschafft. Von den Mönchen durfte nur der offenbar hoch angesehene Abt Mörlin und sein Prior Thomas Carlin bleiben. Beiden wurde sogar gestattet, ihren Mönchshabit weiter zu tragen, und sie durften - da einschlägig eingearbeitet und kompetent - die Verwaltungsarbeit im Kloster weiterführen. In geistlichen Fragen vertraten beide eine konsequente Haltung: Sie wollten von den evangelischen Neuerungen des Herzogs nichts wissen. Mörlin gab das in beeindruckenden Worten zu Protokoll. Man werde nichts hinnehmen, was uns sunst gegen got $[\ldots]$ nit verantwortlich sein mag, $[\ldots]$ sonder vilmer und ehe wir widder ere, trew und aide handeln, ungnad und des elends gewertig sein wollenn. Got verleihe sein gnad und barmherzigkeit. ${ }^{16}$

Obwohl Mörlin und Carlin im Kloster bleiben durften: Zu entscheiden hatten sie nichts mehr, denn Herzog Ulrich hatte im Januar 1536 den Jakob Hofseß aus Heimsheim als Vogt nach Murrhardt geschickt. Er war von nun an fast 40 Jahre lang bis 1574 die entscheidende Person am Ort. Gegen ihn ging nichts. Die Verwaltungstätigkeit von Hofseß lässt sich ziemlich detailliert verfolgen, was an dieser Stelle aus Platzgründen freilich nicht geschehen soll.

Die immensen Geldforderungen Ulrichs - er verlangte, wie erwähnt, allein $60000 \mathrm{fl}$ zur Deckung seiner Kriegskosten bei der Rückgewinnung des Landes - wurden wieder auf die Ämter umgelegt. Angesichts der sowieso schon kritischen Lage der klösterlichen Finanzen war das mit normalen Mitteln nicht mehr zu bewältigen. Das Kloster versuchte sich mit der Verpfändung der Zehnten in

14 Robert Uhland: Herzog Friedrich I. In: Uhland (wie Anm. 13), S. 174-182, hier 178.

15 HStAS A 303, Bd. 10100.

16 Fritz 1990 (wie Anm. 1), S. 108 nach HStAS A 508, Bü 2; der Sachverhalt (wie auch die gesamten Ereignisse von 1534/35) auch bei Werner-Ulrich Deetjen: Die Reformation der Benediktinerklöster Lorch und Murrhardt unter Herzog Ulrich und das „Judicium de votis monasticis“ vom Dezember 1535. In: BWKG 76 (1976), S. 62-115. 
Uttenhofen und Sanzenbach 1534 und in Westheim 1535 an die Stadt Schwäbisch Hall um je 1000 fl über Wasser zu halten. ${ }^{17}$ Der kurzfristige Gewinn der zusammen 2000 fl war mit dem längerfristigen Verlust der zusammen etwa 100 fl jährlicher Einkünfte aus den Zehnten verbunden. Der Zehnt von Uttenhofen und Sanzenbach konnte offenbar wieder eingelöst werden, aber schon 1550 musste das Kloster wieder zu einer Verpfändung schreiten, als ebendiese Zehnten und zusätzlich auch noch die von Bibersfeld an das Stift Komburg verpfändet werden mussten. ${ }^{18}$

Während solche Verpfändungen an der Substanz des Klosterbesitzes kratzten und nur für den Augenblick Abhilfe boten, waren andere Bestrebungen erfolgversprechender: Mit dem 1536 unternommenen Versuch, einen Salzbrunnen zu graben, angeregt durch ettliche burger von Murhartt - also nicht durch den an sich geschäftstüchtigen Mörlin -, wäre eine viel versprechende Wirtschaftspolitik eingeleitet worden. ${ }^{19}$ Leider blieb der Salzversuch erfolglos. Erfolgreicher war die Gründung einer Glashütte in Weidenbach 1550. Aber obwohl dies eine sinnvolle, nachhaltige Wirtschaftspolitik war, brachte sie kurzfristig doch nur geringe Geldbeträge in die Klosterkasse. ${ }^{20}$

Eine tiefe Krise für das evangelische Murrhardt mit dem Vogt und den beiden noch geduldeten Mönchen ergab sich durch den Schmalkaldischen Krieg 1546/47 und die Niederlage der Protestanten. Der siegreiche Kaiser Karl V. führte bekanntlich das Interim ein, jene stark von katholischen Elementen geprägte Zwischenreligion, die in Kraft bleiben sollte, bis das Konzil von Trient die Glaubenseinheit wieder hergestellt hatte.

Für das Kloster Murrhardt bedeutete dies, dass mit dem Neuaufbau eines Konvents begonnen werden konnte. Da Martin Mörlin 1548 starb, oblag es dem zum neuen Abt aufgestiegenen Thomas Carlin, neue Mönche zu gewinnen. Das tat Carlin auch mit einigem Erfolg. Im Verlauf von vier Jahren bildeten fünf junge Mönche, alle um die 20 Jahre alt, den neuen Konvent. Unter den Jungmönchen war auch Otto Leonhard Hofseß, ein Sohn des omnipräsenten Murrhardter Vogts. Der alte Hofseß hatte, mit gutem Gespür für die veränderte religionspolitische Lage, seinen Sohn im Klosterkonvent untergebracht. Außerdem waren 1552 noch drei junge Novizen und zwei alte Mönche vorhanden. Letztere hatten sich nach den Turbulenzen von 1534/35 nicht mehr zurückgewagt und lebten auf Pfarreien bei Würzburg und in Österreich. Unterstützt wurde Carlin von dem als Chronisten bekannten Gelbinger Pfarrer Georg Widman, der seit Jahrzehnten als Notar mit dem Kloster verbunden war und sich dort auch häufig aufhielt.

17 HStAS A 508, Bü 15, 24. Juni (Joh. d. Täufer) 1534 und 19. Juli 1535.

18 HStAS A 508, Bü 15, Verpfändungsurkunde vom 8. Januar 1550.

19 Zum Salzbrunnen: HStAS A 508, Bü 17, verschiedene Schreiben von 1536.

20 Abschrift im Lagerbuch HStAS H 102/54, Bd. 2, fol. 607 ff. 


\section{Wie sehr bewegt die Reformation die einfachen Leute?}

Alles bisher Gesagte steht im Zentrum der Betrachtungen, die durch das 500-Jahr-Jubiläum des Reformationsjahrs 1517 ausgelöst wurden. Ohne Zweifel bestimmten diese Abläufe und Veränderungen das weitere 16. Jahrhundert. Aber die Menschen, die die Quellen zu dieser Zeit verfasst haben (auch zum Kloster Murrhardt), sind mehrheitlich religiöse Akteure - manche, die für die reformatorischen Veränderungen waren, andere, die sich ihnen widersetzten. Indessen würde sich das Bild völlig verzerren, wenn man nur auf die eigentlichen reformatorischen Schritte achtet. Erhebliche Verwerfungen im Verhältnis zwischen Kloster und Bürgern hingen zwar einerseits mit den finanziellen Lasten zusammen, die sich im Umfeld der reformatorischen Entwicklung ergaben, andererseits tauchen diese Verwerfungen sowohl vor als auch nach der Reformation auf. Die Reformation besserte hier nichts.

Mit den Bürgern des Städtchens Murrhardt hatte es schon seit dem Jahr des Bauernkriegs ständige Schwierigkeiten gegeben. 1525 war nach dem Ende des Bauernaufstandes eine neunjährige Steuer auf die Bürger gelegt worden - wobei unklar bleibt, ob wegen deren Verstrickung in den Bauernkrieg oder allgemein, um die bauernkriegsbedingte Schuldenlast des Klosters zu verringern. 1533, ein Jahr vor Ablauf der Abgabe, weigerten sich die Bürger, weiterhin etwas zu zahlen. Die Sache musste vor der habsburgischen Statthalterregierung in Stuttgart verhandelt werden, wo gegen die Bürger entschieden wurde. Das Geld sei 1525 ordentlich bewilligt worden und müsse weiter bezahlt werden. Es wurde sogar entschieden, die jährlich zu Martini fällige Abgabe über die neun Jahre hinaus zu verlängern. ${ }^{21}$ Bemerkenswert ist, dass die 1534/35 von Herzog Ulrich eingeführte Reformation keinerlei Einschnitt in den Spannungen zwischen dem Kloster und den Murrhardter Bürgern brachte. Die Differenzen wegen der städtischen Abgaben dauerten an und eskalierten $1537 \mathrm{zu}$ einem regelrechten Aufstand. Es ging um $200 \mathrm{fl}$, die die Bürger dem Kloster gewährt hatten und die offenbar nicht, wie ausgemacht, zurückbezahlt wurden. ${ }^{22}$ Einzelheiten sind wegen Quellenverlusts zwar nicht zu erkennen, aber die Obrigkeit musste hart vorgehen: Michael Zügel, als Gastwirt offenbar die zentrale Anlaufstation und mutmaßlich Rädelsführer der Unzufriedenen, wurde in Lauffen am Neckar eingesperrt und erst 1538 auf Fürsprache von Mörlin sowie Bürgenstellung wieder freigelassen. ${ }^{23}$

Die Kette der Widersetzlichkeiten der Murrhardter Bürger setzte sich 1550 fort. Als Herzog Ulrich gestorben war, sollten die Untertanen dem neuen Herzog Christoph huldigen. Das war offenbar insofern ein Novum, als man bisher nie

21 HStAS A 508, Bü 16, Konzept, Papierdoppelblatt.

22 Gerhard Fritz: Murrhardter Bürgeraufstände des 16. Jahrhunderts. In: WFr 67 (1983), S. 55-71, hier 57 ff; auch Fritz 1990 (wie Anm. 1), S. 111 f.

23 HStAS A 44, U 2616. 
einem Herzog, sondern immer nur dem Abt gehuldigt hatte. Dies war letztmals 1549 gegenüber dem seit 1548 neu im Amt befindlichen Abt Carlin geschehen. Der konnte 1550 die Lage auch beruhigen und die Untertanen unter Verweis auf seine 1548 mit dem Herzog abgeschlossene Wahlkapitulation aufklären, dass es künftig Huldigungen an den Herzog geben würde. ${ }^{24}$

Aber die Lage blieb labil. Zwar solidarisierten sich die Murrhardter angesichts der seit 1548 auf dem Lande lastenden Besatzung durch die religions- und mentalitätsfremden spanischen Truppen Karls V. zweifellos mit den bekannten einheimischen Inhabern der Macht: Die Spanier hatten nicht nur drückende Versorgungslasten (Wagen mit Heu, später mit Hafer, die nach Winnenden zu liefern waren) gefordert, sondern 1548 durch erhebliche Zerstörungen in Murrhardt auch für schwere Gebäudeschäden gesorgt. Als Carlin 1551 Neubauten errichtete, um die Schäden zu beseitigen, bedeutete dies für die Untertanen erneut schwere Lasten, nicht zuletzt durch Frondienste, zu denen sie bei Baumaßnahmen gegenüber dem Kloster verpflichtet waren. Dazu hin war die materielle Lage der Murrhardter wenig zuvor durch ungünstige klimatische Verhältnisse (hagel unnd mißgewechs) sowieso nicht günstig gewesen. ${ }^{25}$

1564/65 kam es zu einem weiteren Bürgeraufstand, der besser dokumentiert ist als die vorigen. Die meisten Quellen dazu sind erhalten. Es ging vordergründig um die von den Bürgern geforderte Verlesung eines Buchs mit den Bürgerrechten. Eine nicht geringe Rolle spielten darin Fischrechte. Wie schon 1537 endete die Sache auch diesmal mit der Inhaftierung mehrerer Rädelsführer. ${ }^{26}$

Auch ansonsten unterschieden sich die vor- und nachreformatorischen wirtschaftlichen Verhältnisse nicht. Immer wieder gab es in Stadt und Amt Murrhardt neben den genannten Bürgerunruhen Auseinandersetzungen um die knappen landwirtschaftlichen Ressourcen. Die Reihe der inhaltlich stets ähnlichen Konflikte lässt sich bis ins 15. und frühe 16. Jahrhundert zurückverfolgen. ${ }^{27}$

Man muss kein Marxist sein, wenn man in diesem Zusammenhang eine Reihe von marxistisch klingenden Fragen stellt: Was bewegte eigentlich die Murrhardter Bürger mehr? Der ideologisch-religiöse Überbau? Die Ideen Martin Luthers? Der Streit der Theologen? Inwieweit war das für die einfachen Leute im Einzelnen überhaupt nachvollziehbar? Oder war die ökonomische Basis wichtiger? Kam im Sinne Brechts für die Bürger zuerst das Fressen (bzw. das eigene Geld) und dann die (religiöse) Moral? Bewegten die Bürger mehr die handfest zu spürenden finanziellen Folgen der Turbulenzen? Die nach dem Empfinden der Menschen endlos steigenden immer neuen Abgaben und Steuern? Die Finanznot des

24 Zur Huldigungsverweigerung von 1550: Fritz 1990 (wie Anm. 1), S. $128 \mathrm{f}$.

25 So im Brief Carlins an Alexander Demler vom 13. Dezember 1549, HStAS A 87, Bü 16; in diesem Bü und ungefähr zur selben Zeit dort auch die anderen Informationen über die Lieferungen an die Spanier.

26 Fritz 1983 (wie Anm. 22), passim.

27 Gerhard Fritz: Untersuchungen zur Sozial- und Wirtschaftsgeschichte des Schwäbisch-Fränkischen Waldes im 15. und frühen 16. Jahrhundert. In: WFr 96 (2012), S. 59-111. 
Klosters, die mit allen möglichen Maßnahmen auf die Schultern der Bürger abgeladen wurde?

Antworten sind nicht möglich, da - wie erwähnt - die einfachen Bürger und Bauern in den Quellen fast immer stumm bleiben. Wenn sie einmal zu Wort kommen, wie 1556-1558 in einem ausführlich dokumentierten langjährigen Streit um das Retzenholz ostwärts von Fornsbach, dann erscheinen die Obrigkeiten in einer kontinuierlichen Reihe, und es wird in der Wahrnehmung der Akteure nicht zwischen den alten katholischen Äbten und den neuen evangelischen Herren unterschieden. ${ }^{28}$

Die Proteste Luthers gegen die alte Kirche hatten sich entscheidend an der Geldgier Roms entzündet, und weit über Luthers Anhänger im engeren Sinne hinaus war die Ansicht in Deutschland verbreitet, vom Heiligen Stuhl finanziell ausgenommen zu werden. Aber die finanziellen Lasten seit 1525 waren viel schlimmer als alles, was man vorher seitens der alten Kirche erlitten hatte - und all das Geld in den Jahren und Jahrzehnten seit 1525 floss nicht in den Kasten des Papstes, sondern hing mit der Reformation und ihren Verwerfungen und - speziell für Württemberg - mit der beschriebenen chaotischen Politik des Herzogs Ulrich und deren schlimmen Folgen zusammen.

\section{Die zweite Reformation seit 1552}

Das Scheitern der Religionspolitik des Kaisers 1552 fiel zeitlich mit dem Tod Carlins zusammen. Für den reformatorisch gesinnten Herzog Christoph von Württemberg, der 1550 nach dem Tod seines Vaters auf den Thron des Landes gefolgt war, ergab sich damit erstmals die Möglichkeit, eines der im Interim rekatholisierten Klöster abzuwickeln und in evangelischem Sinne umzugestalten. Dabei musste Christoph jedoch behutsam vorgehen, denn über ihm schwebte weiterhin die Drohung des Felonieprozesses. Es war alles zu vermeiden, was dem Kaiser bzw. dessen Bruder, König Ferdinand, Argumente für den Entzug des Herzogtums lieferte.

Deshalb musste Christoph auch im Falle Murrhardts den Anschein erwecken, als führe er dort das alte Benediktinerkloster weiter. Das Doppelspiel, das er dabei betrieb, ist an anderer Stelle ausführlich dargestellt und braucht hier nicht in allen Einzelheiten wiederholt zu werden. Im Innern ließ Christoph durch Erhard Schnepf und den Bietigheimer Vogt Sebastian Hornmold den jungen Konventualen unmissverständlich klarmachen, dass die Zeit des katholischen Benediktinerklosters vorbei war. Nach außen, d. h. gegenüber dem zuständigen Diözesanbischof in Würzburg, wurde die Mimikry ebendieses Klosters weiterhin aufrecht erhalten. So war es nur konsequent, dass Christophs Leute dem Bischof in Würzburg den 19-jährigen Otto Leonhard Hofseß als neuen Abt präsentierten, was der 
Bischof so verständlicherweise nicht akzeptieren wollte: Weder hatte der junge Mann das für einen Abt erforderliche kanonische Alter erreicht noch besaß er die nötige Ausbildung und die nötigen Weihen. Erst wenn er Letzteres nachgeholt hätte, wäre der Bischof möglicherweise einverstanden gewesen. Selbstverständlich war die württembergische Seite nicht bereit, den jungen Hofseß ins katholische Würzburg zu schicken, damit man dort einen anständigen benediktinischen Abt aus ihm machte. Aber die Fiktion sollte aufrecht erhalten werden: Das de facto längst nicht mehr benediktinische Kloster firmierte noch Jahre nach 1552 offiziell als solches - obwohl der Konvent sich (bis auf einen, fürs erste dort verbleibenden Mönch) längst aufgelöst hatte und sich teilweise zu evangelischen Predigern umschulen ließ. Erst als Hofseß junior 1558 heiratete, wurde offensichtlich, dass der neue Abt von Murrhardt nichts mehr mit dem alten Mönchtum zu tun hatte. Anstelle des Konvents waren nun Schüler ins Kloster eingezogen, die in einer Klosterschule unterrichtet wurden.

Damit schien die Reform im Kloster Murrhardt abgeschlossen. Allerdings sollte die Konstellation mit Hofseß senior als Vogt und Hofseß junior als Abt sich als ausgesprochen problembehaftet erweisen. Eigentlich war der Abt der Ranghöhere. Er war in der württembergischen Landschaft vertreten. Der Vogt war „nur“ der Vertreter des Herzogs am Klosterort und eigentlich nur zuständig für die weltliche Verwaltung. Bei einer funktionierenden Konstellation Abt-Vogt kam es zu einer gegenseitigen Kontrolle, die auch durchaus im Sinne des Herzogs war. Nicht zuletzt hatte der Abt die vom Vogt erstellten Amtsrechnungen zu kontrollieren und deren Richtigkeit durch seine Unterschrift zu bestätigen. Es verwundert nicht, dass bei der für Murrhardt spezifischen Vater-Sohn-Konstellation diese Kontrolle problematisch war. ${ }^{29}$

Da man es in Murrhardt mit dieser Konstellation zu tun hatte und da Hofseß senior als erfahrener und durchsetzungsfähiger Verwaltungsfachchef eindeutig der starke Mann war, gab es eine gegenseitige Kontrolle hier nicht, zumal Hofseß junior sich nicht einmal seiner geistlichen Aufgabe gewachsen zeigte, da er sich als ungeeignet zum Predigen erwies. Jahrzehntelang funktionierte das geschlossene „System Hofseß“ gut. 1574 platzte das System. Bei einer Überprüfung der Buchführung war herausgekommen, dass Hofseß senior jahrzehntelang in die eigenen Taschen gewirtschaftet und das Herzogtum um die enorme Summe von 7000 fl betrogen hatte.

Beide Hofse $§$ wurden eingesperrt, Hofse $ß$ senior 1575 nach langwieriger Untersuchung schließlich hingerichtet. Hofseß junior wurde auf dem Hohenneuffen inhaftiert. Da er sich schließlich eher als Opfer seines Vaters und weniger als Täter erwiesen hatte, kam er wieder frei. Seines Amtes enthoben blieb er gleichwohl, wurde aber zu einer Rente begnadigt und starb in Murrhardt 1608 im für die damalige Zeit hohen Alter von 72 Jahren.

29 Vgl. die zahlreichen Unterschriften von Abt Otto Leonhard Hofseß in der von seinem Vater erstellten Amtsrechnung von 1568/69, HStAS A 303, Bd 10110. 
Für Herzog Ludwig, Nachfolger des 1568 verstorbenen Christoph, war die Affäre Hofseß ein schwerer Schlag. ${ }^{30}$ In Murrhardt ließ er durch eine Gruppe besonders loyaler Beamter Remedur machen: Der Renovator Andreas Liesch fertigte 1575/77 für sämtliche Klosterbesitzungen - auch die Außenbesitzungen - „Erneuerungen“" an, d.h. neue Lagerbücher. ${ }^{31}$ Die gesamte Lagerbuchserie von Liesch ist erhalten geblieben und bietet Material für eine noch zu schreibende umfassende Wirtschafts- und Sozialgeschichte des evangelischen Klosters. Mit Zacharias Etzel (1574-1594) wurde ein Mann von untadeligem Ruf neuer evangelischer Abt des Klosters. Und der neue Vogt Gregor Keller (1574-1583/84) wird sich vom Amtsverständnis des alten Hofse $\beta$ ebenfalls fundamental unterschieden haben. Dies aber ist dann bereits die Geschichte des gefestigten Protestantismus und nicht mehr der Umwälzungszeit der Reformation. Deshalb sollen diese innerevangelischen Entwicklungen hier nicht mehr dargestellt werden.

\section{St. Katharina in Hall, das Kloster Murrhardt und die Reformation}

Schon in der Gestalt Georg Widmans gab es eine enge personelle Verflechtung des Klosters Murrhardt mit Schwäbisch Hall. Die Verbindungen des Klosters nach Hall gingen aber weit über Widman hinaus und hatten mit der Haller Kirche St. Katharina auch institutionellen Charakter. Die häufige Anwesenheit der Murrhardter Äbte in der Reichsstadt, wo sie stets standesgemäß mit einigen Kannen Wein begrüßt und bewirtet wurden, unterstreicht die Verbindungen des alten Benediktinerklosters nach Hall. ${ }^{32}$ Um die Zusammenhänge zu verstehen, ist ein Blick auf die Geschichte der Katharinenkirche nötig. Die Katharinenkirche und damit der gesamte Haller Stadtbezirk jenseits Kochens hatten seit etwa fünf Jahrhunderten dem Kloster gehört und waren eine der markantesten Klosterbesitzungen überhaupt gewesen. Aber der kirchenrechtliche Status von St. Katharina war kompliziert. Ursprünglich war St. Katharina eine Filialkirche gewesen.

30 Konrad Rothenhäusler: Die Abteien und Stifte des Herzogthums Württemberg im Zeitalter der Reformation. Stuttgart 1886, S. 136 f, behauptet, die Hofseß-Affäre habe Herzog Christoph schwer getroffen; hier liegt offensichtlich eine Verwechslung vor. Christoph ist bekanntlich 1568 gestorben, die Machenschaften von Hofseß flogen erst 1574 auf.

31 Von den Lagerbüchern sind bisher veröffentlicht: Gerhard Fritz (Hg.): Das Murrhardter Lagerbuch von 1576. Edition des Klosterlagerbuchs von 1576, betreffend die Stadt Murrhardt (Hauptstaatsarchiv Stuttgart H 102/54, Bd. 8). (Schriftenreihe des Instituts für Gesellschaftswissenschaften 1 (elektronische Veröffentlichung). Schwäbisch Gmünd 2010 und Ders. (Hg.): Das Lagerbuch der Murrhardter Weiler von 1575 (Hauptstaatsarchiv Stuttgart H 102/54, Bd. 2). (Schriftenreihe des Instituts für Gesellschaftswissenschaften 7) (elektronische Veröffentlichung). Schwäbisch Gmünd 2017. Zu den Ereignissen um die beiden Hofseß ist eine Veröffentlichung in Vorbereitung.

32 Vgl. zur Bewirtung der Murrhardter Äbte in Hall bis zur Reformation deren Biographien bei Fritz 1990 (wie Anm. 1), S. 325-351. 
Ihre Mutterkirche war die Kirche von Westheim am Kocher, eine der ganz alten, mindestens in karolingische Zeit zurückreichenden Urkirchen in der Gegend. Westheim war 1054 von Kaiser Heinrich III. an das Kloster Murrhardt geschenkt worden. Zu diesem Zeitpunkt bildete im Bereich von Hall offenbar der Kocher die Grenze der Westheimer Kirche, und als dort später (im 12. Jahrhundert?) für die allmählich wachsende Stadt Hall eine kirchliche Versorgung erforderlich wurde, entstand - ergänzend zu St. Michael auf der anderen Seite des Kochers - ein Kirchengebäude. Der noch heute erhaltene Turm der Kirche datiert ins 13., der Chor ins frühe 14. Jahrhundert.

Aufgrund ihrer Bedeutung als städtische Kirche mag St. Katharina bald mehr Pfarrkinder gezählt haben als die alte Mutterkirche in Westheim (von der übrigens im Laufe der Zeit noch weitere Kirchen abgeteilt wurden, namentlich Bibersfeld). Tatsächlich wird schon 1283 ein plebanus St. Catharinae erwähnt, und schon 1403 war St. Katharina seit der Amtszeit von sechs Pfarrern „wie eine rechtmäßige Kirche" geführt worden. Diesen Status mag St. Katharina seit 1354 gehabt haben. In diesem Jahr sei dort nach Widman eine eigene Pfarrei eingerichtet worden. Tatsächlich erfolgte dann 1404 die Trennung der Katharinenkirche von der Westheimer Mutterkirche. Die Murrhardter Rechte an St. Katharina blieben davon unberührt. Das Kloster war weiterhin Patronatsherr (und Visitator eines zumindest zeitweilig existierenden Nonnenklösterchens bei St. Katharina), und die Murrhardter Äbte nutzten ihre Rechte zu häufigen Besuchen in der Reichsstadt. Schon 1512-1514 gab es offenbar Bestrebungen der Haller, das lästige Murrhardter Patronatsrecht loszuwerden, d.h. es selbst zu erwerben, wie man es 1508 schon mit der Kirche St. Michael auf der anderen Seite des Kochers geschafft hatte. ${ }^{33}$ Die Krise des Klosters im ersten und zweiten Jahrzehnt des 16. Jahrhunderts schuf günstige Voraussetzungen für einen Erwerb des Patronatsrechtes. Aber trotz aller Schwierigkeiten änderte sich vorerst an den Verhältnissen nichts. Die Murrhardter legten offenbar Wert darauf, St. Katharina zu behalten, auch wenn - was etwa die Jahrzeitstiftungen angeht - die Verankerung von St. Katharina in Schwäbisch Hall sehr dicht war. Die gesamten Jahrzeitstiftungen weisen keinerlei direkten Bezug zu Murrhardt auf. ${ }^{34}$

Aus der Zeit nach 1521, als der spätere Reformator Michael Gräter vom Kloster die Pfarrei St. Katharina erhalten hatte, liegt ein von den dortigen Pfarrangehörigen an den Rat der Reichsstadt unterzeichneter Brief ${ }^{35}$ vor. Nach einer knappen

33 Zum Vorstehenden Fritz 1990 (wie Anm. 1), S. 266-274; zu den Verhältnissen in Hall auch: Christoph Weismann: Johannes Brenz und die Reformation in Schwäbisch Hall. In: Siegfried Hermle (Hg.): Reformationsgeschichte Württembergs in Porträts. Holzgerlingen 1999, S. 51-73, hier 54, 56, und Hans-Martin Maurer, Kuno Ulshöfer: Johannes Brenz und die Reformation in Württemberg. Stuttgart, Aalen 1988, S. 31-47.

34 Vgl. die Jahrzeitstiftungen von St. Katharina im Stadtarchiv Schwäbisch Hall 4/0139, fol. $437-$ 442.

35 Inwieweit tatsächlich die im Vergleich zur anderen Kocherseite eher armen Pfarrkinder von St. Katharina (,jenhalb Kochens wohnt nichts Rechtes!") den Brief verfasst haben, ist zweifelhaft. Man darf Michael Gräter selbst als geistigen Urheber des Schreibens vermuten. Vgl. zu Gräter: Die Bür- 
Beschreibung des früheren Filialverhältnisses zu Westheim erläuterte das Schreiben, dass Gräters Einkommen von Seiten Murrhardts nur kercklich sei, so dass die Pfarrkinder zur Erhaltung des Pfarrers beitragen müssten, was diese nicht mehr länger tragen wollten. Auch gebe es drei Altarpfründen in St. Katharina. Deren Inhaber lebten aber in Rom und Würzburg, der dritte möglicherweise im thüringischen Arnstadt[?] ${ }^{36}$ und kassierten von ihren Pfründen die jährlich $60 \mathrm{fl}$ Einkünfte wie die Fuckerer. Damit ist entweder gemeint, dass sie das Geld wie das Bankhaus Fugger kassierten, sich also bereicherten - oder aber die Einkünfte waren von den Pfründeninhabern (zur Deckung von Schulden) an die Fugger verpfändet. ${ }^{37}$ Die namentlich nicht genannten Pfründinhaber ließen die Altäre nur durch andere Leute versehen, die lediglich eine spottlich belonung bekämen. Es lag also ein klassischer Fall von Pfründenentfremdung vor, wie er in der vorreformatorischen Zeit immer wieder als besonderes Ärgernis beschrieben wird. Im Falle von St. Katharina hatte offenbar ein päpstlicher, jedenfalls ein in Rom lebender Geistlicher die eine Altarpfründe inne, d.h. faktisch kassierte er nur deren Einkünfte und ließ die Gottesdienste durch einen schlecht besoldeten Vertreter versehen. Ähnlich war die Lage bei den beiden anderen Altarpfründen, wo im einen Fall ein Mann aus dem Umfeld des zuständigen Würzburger Bischofs der Profiteur war, im andern Fall möglicherweise ein Geistlicher aus Arnstadt. Der Brief der Pfarrkinder bat den Rat von Hall darum, sich beim Kloster Murrhardt für ein angemessenes Einkommen Gräters einzusetzen und die Zustände mit den drei Altarpfründen zu beenden. ${ }^{38}$

Es war weniger dem reformatorischen Elan Gräters ${ }^{39}$ zuzuschreiben, als der infolge des Bauernkriegs wieder misslich werdenden finanziellen Lage Murrhardts, dass es bei St. Katharina zu einer endgültigen Lösung kam.

$\mathrm{Zu}$ einem nicht genau bekannten Zeitpunkt 1525 - es heißt nur: in verschinen auffruren, also im zurückliegenden (Bauern-)Krieg - hatte die Stadt Schwäbisch Hall dem Kloster mit einem Kredit von $410 \mathrm{fl}$ unter die Arme gegriffen. Da das Kloster nach den Schäden des Bauernkriegs und den habsburgischen Zahlungsforderungen keinerlei Möglichkeit sah, diese Schuld abzulösen, übertrug es zur Begleichung der $410 \mathrm{fl}$ am 16. Februar 1526 die Haller Katharinenkirche mit-

gerschaft der Reichsstadt Hall von 1395 bis 1600. Bearb. v. Gerd Wunder (Württembergische Geschichtsquellen 25). Stuttgart, Köln 1956, Nr. 2931, S. 275; zum familiären Umfeld Gräters auch: Gerd Wunder: Die Bürger von Hall. Sozialgeschichte einer Reichsstadt 1216-1802 (FWFr 16). Sigmaringen 1980, S. 104.

36 Vgl. unten den Kommentar zur Edition der Urkunde.

37 Ich danke Herta und Wilfried Beutter für den Gedankenaustausch zu dem Fuckerer-Problem der Urkunde.

38 Das undatierte, aber wohl in die Zeit bald nach Gräters Dienstantritt in St. Katharina 1521 anzusetzende Schreiben LKA Stuttgart, A 29/464, 1; das Schreiben ist im Anhang des vorliegenden Beitrags wiedergegeben.

39 Vgl. zu seiner Rolle als Reformator in Hall zusammenfassend: Eduard Krüger: Denkmale aus der Zeit des Humanismus und der Reformation in Schwäbisch Hall. In: WFr 28/29 (1953/54), S. $129-150$, hier S. $149 \mathrm{ff}$. 
samt den dortigen Pfründen und die zu St. Katharina gehörige Kirche mit einer Kaplanei in Sanzenbach sowie sämtlichen daran hängenden Lehenschaften und Einkünften an die Stadt Schwäbisch Hall. ${ }^{40}$ Eine an St. Katharina bestehende Bruderschaft wird in dem Verkauf nicht erwähnt. ${ }^{41}$ Damit endete das fast 500 Jahre alte Engagement Murrhardts in Schwäbisch Hall. Die Reformationsgeschichte von St. Katharina war von diesem Zeitpunkt an identisch mit der Reformationsgeschichte der Reichsstadt, und Michael Gräter spielte in den folgenden Jahrzehnten dort eine herausragende Rolle, die im Rahmen des vorliegenden Beitrags aber nicht weiter zu beleuchten ist.

Aber obgleich die Reichsstadt im Gegensatz zum Kloster Murrhardt, das weiterhin der alten Lehre anhing, seit den 1520er Jahren unter der Ägide von Brenz und Leuten wie Gräter konsequent evangelisch geworden war, darf man sich in dieser Zeit des Übergangs keineswegs einen auf allen Ebenen feindlichen Gegensatz vorstellen. Noch waren die Dinge völlig im Fluss, und wie intensiv und zeitweise geradezu freundschaftlich man miteinander umging, zeigt die Haller Fastnacht von 1533: In dieser frühen Phase der Reformation konnte noch keine Rede von der später für den Protestantismus charakteristischen Faschings-Feindlichkeit sein. Die Reichsstadt lud zu diesem gesellschaftlichen Ereignis auch den Abt von Murrhardt ein. Martin Mörlin kam zum Feiern offenbar gerne. ${ }^{42}$

Weiter murrhardtisch blieb die alte Mutterpfarrei Westheim mit ihrem Filial in Bibersfeld. Die Entscheidungen fielen natürlich nicht in der Murrhardter Pflege Westheim. So ist in den ganzen Reformationsjahrzehnten von dort auch kaum Nennenswertes zu berichten. Erst die Hofseß-Affäre änderte das. Hier steckte der Westheimer Amtmann Hans Koner oder Küener mit Vogt Jakob Hofseß und dessen Betrugsgeschäften unter einer Decke. ${ }^{43}$ Als Hofseß in Stuttgart vernommen wurde, machte man ihm, was Westheim angeht, zum Vorwurf, für die letzten 14 Jahre das Schatzungsgeld in Höhe von $355 \mathrm{fl} 5 \mathrm{x}$ unterschlagen zu haben. Außerdem habe er dortige Weingefälle in Höhe von $160 \mathrm{fl}$ veruntreut und dem Westheimer Schultheißen und einem dortigen Becken einen bzw. einen halben Eimer Wein (Gesamtwert 17 fl $30 \mathrm{x}$ ) unverrechnet zugestellt. ${ }^{44}$

Auch nach dem Ende der Hofseß-Affäre zeigt sich im Hinblick auf die Murrhardter Güter und Rechte in Westheim und den angrenzenden Orten dasselbe Bild wie bei der Pfarrei St. Katharina: Zunächst einmal mussten, wie erwähnt, die Lagerbücher in sämtlichen Orten, an denen das Kloster Besitz hatte, neu angelegt werden, darunter auch Lagerbücher der Pfarreien Westheim, ${ }^{45}$ Bibers-

40 Fritz 1990 (wie Anm. 1), S. 93 f.

41 HStAS H 201, Bd. 116 Gult der bruderschafft zu Sant Katherina von 1527, schmales hochformatiges Heft mit 37 zinsenden Personen, manche mehrfach genannt.

42 Fritz 1990 (wie Anm. 1), S. 98, nach Herolt.

$43 \mathrm{Zu}$ Koner schon erstmals und knapp: Rothenhäusler (wie Anm. 30), S. $136 \mathrm{f}$.

44 HStAS A 508, Bü 14, Vernehmungsprotokoll Hofseß 1574.

45 HStAS H 102/54, Bd. 50 von 1574, enthält auch eine Liste aller Pfarrer von 1559-1792. Er- 
feld ${ }^{46}$ und Fichtenberg ${ }^{47}$ sowie der Pflege Westheim. ${ }^{48}$ Murrhardt und Hall stritten sich über Jahrzehnte um Rechte in Westheim, so $1570-1582,{ }^{49} 1574,{ }^{50} 1578$ $1581^{51}, 1591 .^{52}$ Auch mit Limpurg gab es Klärungsbedarf, da in dem zur Pflege Westheim gehörigen Kondominatsort Ottendorf Rechte strittig waren. 1592 kam es zu einem Vertrag zwischen Württemberg und Limpurg um Malefizrechte in Ottendorf. ${ }^{53} 1617$ musste gar die gesamte Pflege Westheim um $60.000 \mathrm{fl}$ auf zwölf Jahre an Hall verpfändet werden. ${ }^{54}$ Wie im Falle der Katharinenkirche zielte die Politik der Reichsstadt auch in Bezug auf Westheim darauf ab, Murrhardt ganz hinauszudrängen. Dies im Einzelnen weiter darzustellen, ist indessen nicht mehr Thema des vorliegenden Beitrags. ${ }^{55}$

\section{Anhang}

\section{Undatiert, wohl Ende 1521: Schreiben der Pfarrkinder von St. Katharina an den Rat von Schwäbisch Hall}

Ersam weiß, gúnstig herrn, $e$ [wer] $e[\mathrm{r}] w[$ urden] seyen unser underthenig schuldig diennst zuvor.

Gunstigen herren! Der erwirdig herr und geistlich vatter, herr Oßwaldt, appt unnd convent zu Murhart, haben unns zu geschickt her Micheln Grettern, die pfarr kirchen zu sant Katherinen und uns als pfarrkinder hie zu Hall jennenhalbs Kochens mit pfarlichen ämptern zu versehen, an welchem wir mit danckparem gemút beniegig, und die genannt pfarkirchen und folck als pfarkúndern ettwan von der pfarr zú Weschthain als ein dochter eingeleibt, welche dan zur zeit von gemainen guett unnd einkomens der obern pfarrkirchen und pfarhers zu Weschtaim wúrde versehen, mit sampt nuzung und arbait ain ander angeherig.

wähnt sei auch das Westheimer Lagerbuch von 1539, das einzige erhaltene Lagerbuch aus der Zeit des Benediktinerklosters, ebd. Bd. 48.

46 HStAS H 102/54, Bd. 54 von 1575; der auf den 17. August 1573 datierte Bd. 49 ist ein recht nachlässig geführtes Konzept mit fliegenden Blättern.

47 HStAS H 102/54, Bd. 5 von 1575.

48 HStAS H 102/54, Bd. 51 von 1575, Bd. 60 zur Pfarr Westheim über Öttendorff und Niderndorff von 1582.

49 HStAS A 508, Bü 9, wegen der Obrigkeit in Westheim und wegen der Novalien.

50 HStAS A 508, Bü 9, Klag-Libellen und Rezesse wegen der Pflege Westheim.

51 HStAS A 508, Bü 17, umfangreiche Streitigkeiten zwischen 1578-1591; zu 1578 auch Bü 6.

52 HStAS A 508, Bü 10, Streit über Geleit und Holzfällen in der Pflege Westheim.

53 Extrakt aus dem Vertrag HStAS H 102/54, Bd. 60, fol. I-II. Die Gerichtsbarkeit sollte bei Malefizfällen zwischen Württemberg und Limpurg abwechseln. Gerichtsstand sollte Marbach bzw. Gaildorf sein.

54 HStAS A 508, Bü. 11.

55 Vgl. dazu: Manfred Akermann: Westheim unter der Herrschaft von Hall und Württemberg - das Kondominat von der Reformation bis 1802. In: Gottfried Bazlen (Hg.): Westheim am Kocher. 1200 Jahre Geschichte. Rosengarten-Westheim 1988, S. 71-85, hier $71 \mathrm{ff}$. 
Aber dieweil die genanten apt und convent zu Murhart us $\beta$ gaistlichem genaigtem grunt erbetten unnd erworben, das durch seiner gnaden und convent pitte die pfarkirch zu sanct Katherinen mit sampt angeherigem folck von der gemainen pfarkirchen zu Weschtaim abgeschaiden, ain besondere ewige vicarj und pfarre gemacht, welche den genanten heren appt und convent zu Murhart zu ewigem lehen oder presentation vorbehalten.

Dweil die múhe, sorg und pferliche arbait uff genante pfarrkirchen sannct Katherinen erwachsen, das einkumens, so ainem pfarrherrn gepúrlich und göttlich zúgeherig, leibs notturfft zu erhalten, kercklich bedacht worden, jezundt uff unns, die wir verarmpt erwachßen, pfärliche beschwerde und innkomens, kennen und megen wir solliche beschwerdt nit lenger gedulden, dan wir súnst mit búrgerlichen beschwerden, steuer und bettgelden beladen.

Auch sein drey altaren in der gemainen kirchen sanct Katherinen, welche den genanten erwirdigen herren appt unnd convent zu Murhart zu lehen gen, deren besizer zu Rom, Wúrzpurg unnd Amstat [s. u.] Fuckerer, jerlich ubernuzung nemen bey sechzig gulden, verstecken die altaren mit andern bepfrúnd personnen umb spottlich belonung, lassen die pfründen heusser auß unbúhr zu nichten werden, welchs der pfarrkirchen verclainung unnd unachtung bringt unnd verderbung, so sollich wechselbenck lennger mit der armen kirchen muttwillen wurden.

Darumb, // ersamen, weißen, gunstigen lieben herren, bitten wie armen underthenfleissig umb gottes willen $e$ [wer] $e[\mathrm{r}] w$ [urden] welle unser beschwerten underthon gnediglich bedencken mit furschrifft unnd bitt an gedachten erwirdigen herrn appt und convent zu Murhart.

Verhoffen wir, sein erwirde werden us $\beta$ geistlichem cristlichem gemút unns $e$ [wer] $e[\mathrm{r}] w$ [urden] furbit lassen geniessen umb gottes willen begnaden unnd unserm pfarherrn on unser beschwerdt und schazung ein geburlichs, erbers einkomens, von dem er notturftig enthaltung haben mege und der dreyer altar núzung in besserung verordnet werde unnd unser pfarrer in gottes diennst unns zu frúmen geflissen sey, wellen wir mit unnserm gebet zu dem allmechtigen unnd armen schuldigen diennsten alzeit berait sein, in williger weis zuverdienen, pitten unndertheniglich umb gnedig antwurt.

$E[\mathrm{wer}] e[\mathrm{r}] w[\mathrm{urden}]$ unnderthon gannze gemain pfarkind zu sanct Katherinen jenhalbs Kochenns.

(LKA Stuttgart, A 29/464, 1, undatiert; auf Umschlag in späterer Schrift: Hall. Pfarkünder zu sanct Catharinen bitten den rath umb zueschrifft ad abbatem Murhartensem, so ihnen einen pfarher gesandt und zu der pfar Westheim separirt. Petunt parocho sustentat ex beneficijs. 
Oben ist Groß- und Kleinschreibung normalisiert, vokalisches v in u umgewandelt, konsonantisches u in v. Interpunktion ergänzt. Zeileneinteilung und Absätze wurden oben - abweichend vom durchgehend geschriebenen Original - der Klarheit halber eingefügt. Jeweils am Zeilenende sind wegen Textverlusts einzelne Buchstaben nicht mehr zu lesen, aber leicht zu ergänzen, was oben stillschweigend vorgenommen wurde. Abkürzungen werden mit [eckigen] Klammern markiert. // markiert das Seitenende des im Original auf zwei Seiten geschriebenen Textes).

Zu Amstat: Der Text liest Amstat, nicht Arnstat. Sollte es sich um eine Verschreibung $-m$ - statt $-r n$ - handeln, würde es sich um Arnstadt handeln, da es einen Ort „Amstadt“ nicht gibt. In Arnstadt waren mit zwei Klöstern plus Weltgeistlichen auch genügend potentielle Pfründinhaber vorhanden. Die beiden Orte namens „Amstetten“ wären sprachlich u. U. ebenfalls denkbar, aber von der Sache her unwahrscheinlich: Amstetten (Alb-Donau-Kreis) besaß als kleines Dorf wohl keinen geeigneten Geistlichen, der in Frage käme, Amstetten (Niederösterreich) läge geographisch sehr abseits und lässt keine irgendwie geartete Verbindung zu Murrhardt erkennen. Nicht ganz auszuschließen ist aber auch, dass es sich nicht um einen Ortsnamen handelt, sondern dass amstat soviel heißt wie ,an Stelle von“" bzw. ,anstatt“. Dann wäre das Ganze so zu verstehen, dass die drei Pfründinhaber, die dann nur in Rom und Würzburg säßen, das Geld scheffelten „wie die Fugger". 